\title{
The need of standardization and of large clinical studies in an emerging indication of $\left[{ }^{18}\right.$ F]FDG PET: the autoimmune encephalitis
}

\author{
Silvia Morbelli ${ }^{1} \cdot$ Javier Arbizu $^{2} \cdot$ Jan Booij $^{3} \cdot$ Ming-Kai Chen $^{4} \cdot$ Gael Chetelat $^{5,6,7,8}$. $^{1}$ \\ Donna J. Cross $^{9}$ • Mehdi Djekidel ${ }^{10,11}$ • Alexander Drzezga ${ }^{12}$ • Ozgul Ekmekcioglu ${ }^{13}$. \\ Valentina Garibotto $^{14}$ - Swen Hesse ${ }^{15}$ - Kazunari Ishii ${ }^{16} \cdot$ Lida Jafari Saraf $^{17}$. \\ Adriaan A. Lammertsma ${ }^{18}$ - Ian Law $^{19}$ - Dana Mathews ${ }^{20}$ - Satoshi Minoshima ${ }^{9}$

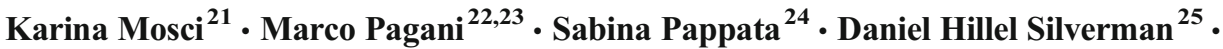 \\ Alberto Signore $^{26}$ • Elsmarieke Van De Giessen ${ }^{3}$ • Victor Villemagne Vi,28,29 $^{\text {. }}$ \\ Henryk Barthel ${ }^{15}$ on behalf of the European Association of Nuclear Medicine (EANM) \\ and of the Society of Nuclear Medicine and Molecular Imaging (SNMMI)
}

Received: 24 November 2016 / Accepted: 24 November 2016/Published online: 6 December 2016

(C) Springer-Verlag Berlin Heidelberg 2016

\section{Introduction}

$\left[{ }^{18} \mathrm{~F}\right]$ FDG PET is currently the most commonly used functional imaging method for the in vivo investigation of regional brain metabolism in clinical practice. PET/CT scanners are widely available in Europe, in the US, in Australia, and most of the rest of the world [1] and the clinical role of $\left[{ }^{18} \mathrm{~F}\right] \mathrm{FDG}$ brain PET can be regarded as established for a number of diagnostic challenges in Neurology and Psychiatry [2]. In particular, $\left[{ }^{18} \mathrm{~F}\right]$ FDG PET

Silvia Morbelli

silviadaniela.morbelli@hsanmartino.it

on behalf of the European Association of Nuclear Medicine (EANM) and of the Society of Nuclear Medicine and Molecular Imaging (SNMMI)

1 Nuclear Medicine, Department of Health Science (DISSAL), University of Genoa and IRCCS AOU San Martino-IST, Largo R. Benzi 10, 16132 Genoa, Italy

2 Department of Nuclear Medicine, Clinica Universidad de Navarra, University of Navarra, Pamplona, Spain

3 Department of Nuclear Medicine, Academic Medical Center, University of Amsterdam, Amsterdam, The Netherlands

4 Radiology and Biomedical Imaging, Yale University School of Medicine, New Haven, CT, USA

5 Inserm, U1077, Caen, France

6 Université de Caen Basse-Normandie, UMR-S1077, Caen, France plays a major role in the early and differential diagnosis of neurodegenerative dementias and Parkinsonian syndromes by showing disease-specific patterns of hypometabolism [3]. For a substantial period of time, however, the lack of standardisation in reading images and reporting results, as well as the lack of large studies in homogeneous patient cohorts, has delayed a wider routine clinical use of $\left[{ }^{18} \mathrm{~F}\right]$ FDG PET [4]. This is particularly true in the field of inflammation/infection imaging despite initial attempts

7 Ecole Pratique des Hautes Etudes, UMR-S1077, Caen, France

8 CHU de Caen, U1077, Caen, France

9 Department of Radiology and Imaging Sciences, University of Utah, Salt Lake City, UT, USA

10 University of Michigan Ann Arbor, Hamden, Michigan, USA

11 QMC-Quality Medical Consulting, Hamden, Michigan, USA

12 Department of Nuclear Medicine, University of Cologne, Cologne, Germany

13 Department of Nuclear Medicine, Bagcilar Education and Research Hospital, Istanbul, Turkey

14 Department of Medical Imaging, Geneva University and Geneva University Hospitals, Geneva, Switzerland

15 Department of Nuclear Medicine, University of Leipzig, Leipzig, Germany

16 Department of Radiology, Faculty of Medicines, Kindai University, Osaka, Japan 
to standardize this methodology between the European Association of Nuclear Medicine (EANM) and the American Society of Nuclear medicine and Molecular Imaging (SNMMI) [5]. In the last decade, thanks to the synergistic efforts of the neuroimaging and neurological communities across different countries, larger data sets and functional imaging repositories have become available. This allowed for assessing the clinical value of $\left[{ }^{18} \mathrm{~F}\right]$ FDG PET as well as that of other imaging biomarkers in the field of neurodegenerative diseases [6-8]. The added value of this large multi-center approach has gained attention in the US for PET imaging in other indications as well, for example, through the National Oncologic PET Registry (NOPR) and more recently the Imaging Dementia Evidence for Amyloid Scanning (IDEAS) clinical trials. Similarly, software based semi-quantitative approaches have increasingly been used both in the context of published group analyses as well as in clinical practice, thus testifying that objective measures can be incorporated and added to the traditional analysis approach in nuclear medicine, i.e., visual reading [9-11].

Accordingly, the long and rough path needed to develop the proper methodologies for clinical validation of $\left[{ }^{18} \mathrm{~F}\right]$ FDG PET in neurodegenerative diseases now allows

\footnotetext{
17 Department of Radiology, Veterans Affairs Healthcare System, Greater Los Angeles, CA, USA

18 Department of Radiology \& Nuclear Medicine, VU University Medical Center, Amsterdam, The Netherlands

19 Department of Clinical Physiology, Nuclear Medicine and PET, Copenhagen University Hospital, Rigshospitalet, Copenhagen, Denmark

20 Department of Radiology, University of Texas Southwestern Medical Center, Dallas, TX, USA

21 Departament of Nuclear Medicine, Hospital das Forças Armadas, Brasilia, Brazil

22 Institute of Cognitive Sciences and Technologies, CNR, Rome, Italy

23 Department of Nuclear Medicine, Karolinska Hospital, Stockholm, Sweden

24 Institute of Biostructure and Bioimaging, CNR, Naples, Italy

25 Department of Molecular and Medical Pharmacology, University of California, Los Angeles, CA, USA

26 Nuclear Medicine Unit, Department of Medical-Surgical Sciences and of Translational Medicine, "Sapienza" University of Rome, Rome, Italy

27 Department of Molecular Imaging, Austin Health, Melbourne, Australia

28 Department of Medicine, University of Melbourne, Melbourne, Australia

29 Florey Institute of Neuroscience and Mental Health, University of Melbourne, Melbourne, Australia
}

for an accelerated process of understanding and validating new emerging indications of this functional imaging tool.

\section{The autoimmune encephalitis}

A relevant example is autoimmune encephalitis (AE), an emerging clinical and treatable entity, for which the diagnostic approach is, at present, under revision with the aim of performing earlier diagnosis of possible $\mathrm{AE}$ even before the availability of autoantibody testing results [12].

In the past ten years, the discovery of several autoantibodies specific for the neuronal cell membrane surface or synaptic proteins has led to the emerging recognition of clinical entities belonging to the spectrum of $\mathrm{AE}$ [12]. Patients with $\mathrm{AE}$ may share core symptoms of infectious encephalitis but could also present with memory or behavioral deficits, without fever, or with alteration in the level of consciousness. Autoantibody testing is crucial for the differential diagnosis. As a drawback, it is not accessible in many institutions and for technical reasons results are not readily available thus delaying diagnosis and the initiation of an effective treatment [12]. On the other hand, it has been demonstrated that early initiation of immunosuppressive therapy may result in improved clinical outcome [13].

In a position paper recently published in Lancet Neurology, Graus and colleagues propose a new syndrome-based diagnostic approach to $\mathrm{AE}$ with the aim of allowing an earlier diagnosis of possible $\mathrm{AE}$ thus leading to prompt immunotherapy initiation [12]. According to this newly proposed approach, the early diagnostic work-up should rely on conventional neurological evaluation and standard diagnostic tests (i.e., MRI, cerebrospinal-fluid, or EEG). Comprehensive antibody tests would then be needed to substantiate the clinical diagnosis (probable or definite AE) and to refine treatment. This newly proposed approach follows the identification of an increasing number of non-infectious types of $\mathrm{AE}$ and supports clinicians in the evaluation of acute or subacute presentations that do not meet existing criteria. For the diagnosis of possible AE, three criteria need to be fulfilled: (1) reasonable exclusion of other causes, (2) subacute onset with working memory deficits, altered mental status or psychiatric symptoms, and (3) at least one of the following: new focal CNS findings, seizures that cannot be explained otherwise, CSF pleocytosis, or MRI suggestive of encephalitis. After the discussion of the initial clinical assessment of possible causes of encephalitis, Graus et al. restricted their discussion to AE showing specific features (limbic encephalitis, anti-NMDA receptor encephalitis, Bickerstaff's brainstem encephalitis, acute disseminated encephalomyelitis and Hashimoto's encephalopathy). In this framework, the presence of MRI abnormalities (T2-weighted fluid-attenuated inversion recovery alterations highly restricted to the medial temporal lobes and possibly bilateral) is listed among the criteria for the diagnosis of autoimmune limbic encephalitis. MRI is obviously 
mandatory and represents a first level imaging study in these patients. However, as the authors acknowledge, limbic encephalitis can occur with normal MRI findings or with MRI evidence of unilateral involvement only [12].

\section{Why $\left[{ }^{18}\right.$ F]FDG in AE?}

It has been demonstrated that sensitivity of $\mathrm{AE}$ diagnosis can be significantly increased by identifying medial temporal lobe hypermetabolism (or sometimes hypometabolism) on $\left[{ }^{18} \mathrm{~F}\right] \mathrm{FDG}$ PET even in temporal lobe structures with normal (or unilaterally altered) MRI signal [14-16]. However, the potential role of $\left[{ }^{18}\right.$ F]FDG PET in the syndrome-based approach to AE has been somewhat downplayed in the position paper by Graus and colleagues. According to the authors, two reasons underlie this limited recognition [17]. First, the lack of prompt availability of this method for indications needing an emergency-basis disposability. Second, the need for further studies to validate its predictive value for the diagnosis of $\mathrm{AE}$ [17]. On the one hand, we might argue that the number of PET/CT scanners available in industrialized countries and the relative short scanner occupancy will allow the prompt availability of this method for patients with suspected AE once its clinical use for this indication gains traction and becomes mainstream. On the other hand, we agree that larger validation studies are needed. As previously and presently done in the field of neurodegenerative disorders, the nuclear medicine imaging community should work to help clinicians find solutions for unmet diagnostic needs in patients with AE. Accordingly, we would like to take the opportunity, following our response to the Gauss et al. paper in The Lancet Neurology [16], to further discuss the main available lines of evidence on the potential role of $\left[{ }^{18} \mathrm{~F}\right] \mathrm{FDG}$ PET in $\mathrm{AE}$, to delineate challenges, and to suggest the path for future collaborative efforts with the aim of collecting multi-modal biomarker databases in AE patients including $\left[{ }^{18} \mathrm{~F}\right] \mathrm{FDG}$ PET data.

Although often reported within the context of case series, an increasing amount of literature showed that $\left[{ }^{18} \mathrm{~F}\right] \mathrm{FDG}$ PET was a reasonable means of enhancing the sensitivity of the diagnostic algorithm of AE as PET can show medial temporal lobe hypo- or hypermetabolism even in normally appearing temporal lobe structures on MRI [14, 15]. Moreover, it has been reported that, both in limbic encephalitis and in other AE subtypes, $\left[{ }^{18}\right.$ F]FDG PET can show extra-limbic abnormalities (more often hypermetabolism), e.g., in the brainstem, cerebellum or cerebral cortex, and PET findings seem to correlate more closely with clinical symptoms than MRI findings [18]. In this scenario, a more frequent association between autoantibodies against intracellular antigens and mesiotemporal abnormalities has been suggested, whilst autoantibodies against surface antigens seem to be more often associated with abnormalities outside the mesiotemporal region $[14$, 15]. Extra-limbic hypermetabolism can be relevant for diagnostic purposes and these features can be used in the future to investigate the different pathophysiological mechanisms underlying limbic encephalitis (possibly linked to different autoantibodies) [14-19].

The diagnosis of possible encephalitis with antibodies against the N-methyl-D-aspartate receptor (NMDAR) is also a great challenge and criteria for its identification have been addressed, too [12]. MRI is not included in these criteria as in large cohort studies MRI abnormalities have been reported in only $23-50 \%$ of patients [20,21]. Further investigations would be advisable to specifically evaluate the role of $\left[{ }^{18} \mathrm{~F}\right] \mathrm{FDG}$ PET in NMDAR encephalitis. In fact, it has been suggested that $\left[{ }^{18} \mathrm{~F}\right] \mathrm{FDG}$ PET can reveal pathological changes in NMDAR encephalitis even when MRI and CT scans are normal. $\left[{ }^{18} \mathrm{~F}\right]$ FDG PET was altered in at least $50 \%$ of cases with normal MRI in the small groups of patients with NMDAR encephalitis studied with both imaging modalities [20,22]. Metabolic abnormalities (hypo- or hypermetabolism) have been observed throughout the brain, including the frontal, temporal, and occipital lobes as well as basal ganglia, cerebellum, and brainstem [14]. Again, PET findings have been more clearly associated with the clinical picture, disease severity, and recovery after therapy than MRI findings [14]. This evidence is in accordance with the well-known general high sensitivity of $\left[{ }^{18} \mathrm{~F}\right] \mathrm{FDG}$ PET in correlating and predicting the clinical course of disease [23] as well as the response to therapy. Accordingly, a potential role of $\left[{ }^{18} \mathrm{~F}\right]$ FDG PET should be further addressed also in patients with suspected disease relapse of NMDAR encephalitis (which can occur in around $10 \%$ of patients), [21]. Moreover, PET imaging of ion channel-linked receptors is also under development and tracers for imaging NMDAR activity have been evaluated already in preclinical studies and healthy humans [24]. The potential role of these tracers should be tested in the future also in patients with AE.

As a general remark, specific challenges are present in the clinical assessment of $\mathrm{AE}$ in elderly people given the possible presence of age-related brain abnormalities as well as to the coexistence of other disorders affecting memory and cognition. Especially in case of inconclusive MRI findings, $\left[{ }^{18} \mathrm{~F}\right] \mathrm{FDG}$ PET can be performed in elderly patients with clinical suspicion of AE. In these patients, $\left[{ }^{18} \mathrm{~F}\right]$ FDG PET can highlight a pattern of metabolic changes typical of other diseases thus helping in the differential diagnosis and guiding the diagnostic work-up [3].

Finally, both in elderly and younger patients a whole-body $\left[{ }^{18} \mathrm{~F}\right]$ FDG PET scan is often added to the paraneoplastic workup of AE when screening patients for malignancy (as PET/CT is more sensitive than CT alone), [14]. As it adds no more than $10 \mathrm{~min}$ to the total examination time, a dedicated brain scan can easily be included to the same PET session, and, if needed, can also be used to monitor response to therapy or used in the context of suspected disease relapse [19].

A multimodality imaging approach to $\mathrm{AE}$ can also be of particular interest given the recent availability of combined PET and MRI systems. Hybrid PET/MRI systems are 
presently tested in the diagnostic algorithm of other brain disorders [25] and in the future, can emerge as a suitable way to investigate $\mathrm{AE}$ both from the diagnostic and pathophysiologic points of view [26]. In this context the recent implementation of advanced algorithms for attenuation correction $[25,27]$ can be of particular interest in patients with frequent MRI abnormalities at the mesial temporal lobe level such as in the presence of limbic encephalitis.

Although $\left[{ }^{18} \mathrm{~F}\right]$ FDG PET is a sensitive and widely available imaging method, some intrinsic challenges related to the characteristics of the tracer employed as well as some specific issues should be systematically addressed when validating it as a diagnostic tool for $\mathrm{AE}$.

In fact, $\left[{ }^{18} \mathrm{~F}\right] \mathrm{FDG}$ PET has the ability to estimate the local cerebral metabolic rate of glucose, thus providing information on the synapse function and dysfunction in vivo [28]. Although this feature is the source of the high sensitivity and wide-range clinical use of this tool, it also underlies a lack of specificity. Indeed, the presence of reduced $\left[{ }^{18} \mathrm{~F}\right] \mathrm{FDG}$ PET uptake may be due to a variety of underlying pathological processes (i.e., neuronal dysfunction, atrophy, vascular damage) and in the field of neurodegenerative disorders specific hypometabolic patterns are recognized for different diseases. The identification of specific disease patterns should be undertaken also in AE patients with some peculiar challenges. In fact, both areas of hyper- and hypometabolism may coexist in these patients, and the etiological diagnosis of hypermetabolism can even be more challenging, as patients with encephalitis (either infectious or noninfectious) can present with areas of hypermetabolism.

Although visual analysis is obviously the first step of brain $\left[{ }^{18} \mathrm{~F}\right]$ FDG PET reading, in clinical practice the lack of expertise and the lack of objective semi-quantitative measures may still prevent the recognition of the actual (and concurrent with patient's clinical presentation) disease pattern. As a drawback, some (semi)automated approaches to analyse $\left[{ }^{18} \mathrm{~F}\right] \mathrm{FDG}$ PET data are not suitable for identifying areas of hypermetabolism, as they were developed for diagnosing hypometabolic patterns in Alzheimer's disease [9]. Conversely, some voxelbased approaches might highlight areas of relative hypermetabolism just as a result of bias introduced by intensity normalization procedures and thus the choice of the reference region for intensity normalization will be crucial in this setting [29]. Moreover, it is worthwhile to mention that absolute quantification of brain glucose consumption can also be performed by means of $\left[{ }^{18} \mathrm{~F}\right] \mathrm{FDG}$ PET, thus potentially allowing a pathophysiological validation of the hypermetabolic, hypometabolic, and/or mixed patterns in the context of prospective research trials in $\mathrm{AE}$ patients.

As a final remark, the presence of distinct metabolic patterns among the various $\mathrm{AE}$ subtypes might be a further source of trouble in group analyses. Only the availability of larger subgroups of AE patients may allow for uncovering $\mathrm{AE}$ subtype-specific diagnostic potentials of $\left[{ }^{18} \mathrm{~F}\right] \mathrm{FDG}$ PET, as previously done for heterogeneous patient-based patterns of epilepsy [30]. Taking together, the clinical community of the field has just provided a crucial contribution to pave the way for an early diagnosis of AE $[12,16]$. The next step will be to test the new proposed criteria for sensitivity and specificity in clinical practice [31]. This should also be an opportunity to assess the possible contribution of $\left[{ }^{18} \mathrm{~F}\right]$ FDG PET within the general diagnostic workup of $\mathrm{AE}$ as well as in selected scenarios. In this framework, the Nuclear Medicine community should work in a joint effort of data sharing with the aim of quickly translating the available scientific evidence into standard procedures and accelerating the comprehension and validation of $\left[{ }^{18} \mathrm{~F}\right] \mathrm{FDG}$ PET in the emerging field of AE.

\section{Compliance with Ethical Standards}

Conflict of Interest The authors declare that they have no conflict of interest.

This article does not contain any studies with human participants or animals performed by any of the authors.

\section{References}

1. National Research Council (US) and Institute of Medicine (US) Committee on State of the Science of Nuclear Medicine. Advancing Nuclear Medicine Through Innovation, Washington (DC): National Academies Press (US); ISBN-13: 978-0-30911067-9, ISBN-10: 0-309-11067-X; 2007.

2. Varrone A, Asenbaum S, Vander Borght T, Booij J, Nobili F, Någren K, et al. EANM procedure guidelines for PET brain imaging using [18F]FDG, version 2. Eur J Nucl Med Mol Imaging. 2009;36:210310.

3. Herholz K. Guidance for reading FDG PET scans in dementia patients. Q J Nucl Med Mol Imaging. 2014;58:332-43.

4. Morbelli S, Garibotto V, Van De Giessen E, Arbizu J, Chételat G, Drezgza A, et al. A cochrane review on brain $\left[{ }^{18} \mathrm{~F}\right]$ FDG PET in dementia: limitations and future perspectives. Eur J Nucl Med Mol Imaging. 2015;42:1487-91.

5. Jamar F, Buscombe J, Chiti A, Christian PE, Delbeke D, Donohoe $\mathrm{KJ}$, et al. EANM/SNMMI guideline for 18F-FDG use in inflammation and infection. J Nucl Med. 2013;54:647-58.

6. Hendrix JA, Finger B, Weiner MW, Frisoni GB, Iwatsubo T, Rowe $\mathrm{CC}$, et al. The worldwide Alzheimer's disease neuroimaging initiative: an update. Alzheimers Dement. 2015;11:850-9.

7. Weiner MW, Veitch DP, Aisen PS, Beckett LA, Cairns NJ, Cedarbaum J, et al. 2014 update of the Alzheimer's disease neuroimaging initiative: a review of papers published since its inception. Alzheimers Dement. 2015;11:e1-120.

8. Ellis KA, Bush AI, Darby D, De Fazio D, Foster J, Hudson P, et al. The Australian Imaging, Biomarkers and Lifestyle (AIBL) study of aging: methodology and baseline characteristics of 1112 individuals recruited for a longitudinal study of Alzheimer's disease. Int Psychogeriatr. 2009;21:672-87.

9. Herholz K, Westwood S, Haense C, Dunn G. Evaluation of a calibrated (18)F-FDG PET score as a biomarker for progression in Alzheimer disease and mild cognitive impairment. J Nucl Med. 2011;52:1218-26. 
10. Perani D, Della Rosa PA, Cerami C, Gallivanone F, Fallanca F, Vanoli EG, et al. Validation of an optimized SPM procedure for FDG-PET in dementia diagnosis in a clinical setting. Neuroimage Clin. 2014;6:445-54.

11. Morbelli S, Brugnolo A, Bossert I, Buschiazzo A, Frisoni GB, Galluzzi S, et al. Visual versus semi-quantitative analysis of $18 \mathrm{~F}-$ FDG-PET in amnestic MCI: an European Alzheimer's Disease Consortium (EADC) project. J Alzheimers Dis. 2015;44:815-26.

12. Graus F, Titulaer MJ, Balu R, et al. A clinical approach to diagnosis of autoimmune encephalitis. Lancet Neurol. 2016;15:391-404.

13. Vollmer TL, McCarthy M. Autoimmune encephalitis: a more treatable tragedy if diagnosed early. Neurology. 2016;86(18):1655-6.

14. Heine J, Prüss H, Bartsch T, Ploner CJ, Paul F, Finke C. Imaging of autoimmune encephalitis - relevance for clinical practice and hippocampal function. Neuroscience. 2015;309:68-83.

15. Baumgartner A, Rauer S, Mader I, Meyer PT. Cerebral FDG-PET and MRI findings in autoimmune limbic encephalitis: correlation with autoantibody types. J Neurol. 2013;260:2744-53.

16. Morbelli S, Djekidel M, Hesse S, Pagani M, Barthel H, Neuroimaging Committee of the European Association of Nuclear Medicine (EANM); Brain Imaging Council of the Society of Nuclear Medicine and Molecular Imaging (SNMMI). Role of (18)F-FDG-PET imaging in the diagnosis of autoimmune encephalitis. Lancet Neurol. 2016;15:1009-10.

17. Graus F, Dalmau J. Role of (18)F-FDG-PET imaging in the diagnosis of autoimmune encephalitis - authors' reply. Lancet Neurol. 2016;15:1010.

18. Ances BM, Vitaliani R, Taylor RA, Liebeskind DS, Voloschin A, Houghton DJ, et al. Treatment-responsive limbic encephalitis identified by neuropil antibodies: MRI and PET correlates. Brain. 2005;128:1764-77.

19. Trevino-Peinado C, Arbizu J, Irimia P, Riverol M, Martínez-Vila E. Monitoring the effect of immunotherapy in autoimmune limbic encephalitis using [18F]FDG PET. Clin Nucl Med. 2015;40:e441-3.

20. Irani SR, Bera K, Waters P, Zuliani L, Maxwell S, Zandi MS, et al. $\mathrm{N}$-methyl-D-aspartate antibody encephalitis: temporal progression of clinical and paraclinical observations in a predominantly nonparaneoplastic disorder of both sexes. Brain. 2010;133:1655-67.
21. Titulaer MJ, McCracken L, Gabilondo I, Armangué T, Glaser C, Iizuka $\mathrm{T}$, et al. Treatment and prognostic factors for long-term outcome in patients with anti-NMDA receptor encephalitis: an observational cohort study. Lancet Neurol. 2013;12:157-65.

22. Wegner F, Wilke F, Raab P, Ben Tayeb S, Boeck A, Haense C, et al. Anti-leucine rich glioma inactivated 1 protein and anti-N-methylD-aspartate receptor encephalitis show distinct patterns of brain glucose metabolism in 18F-fluoro-2-deoxy-D-glucose positron emission tomography. BMC Neurol. 2014;14:136.

23. Chételat G, Desgranges B, De la Sayette V, Viadre F, Eustache F, Baron JC. Mild cognitive impairment: can FDG-PET predict who is to rapidly convert to Alzheimer's disease? Neurology. 2003;60:1374-7.

24. van der Doef TF, Golla SS, Klein PJ, Oropeza-Seguias GM, Schuit RC, Metaxas A, et al. Quantification of the novel N-methyl-daspartate receptor ligand $[11 \mathrm{C}] \mathrm{GMOM}$ in man. J Cereb Blood Flow Metab. 2016;36:1111-21.

25. Bailey DL, Pichler BJ, Gückel B, Barthel H, Beer AJ, Botnar R, et al. Combined PET/MRI: from status quo to status go. Summary report of the Fifth International Workshop on PET/MR imaging. Mol Imaging Biol. 2016;18(5):637-50.

26. Fayad H, Lamare F, Merlin T, Visvikis D. Motion correction using anatomical information in PET/CT and PET/MR hybrid imaging. Q J Nucl Med Mol Imaging. 2016;60(1):12-24.

27. Chen KT, Izquierdo-Garcia D, Poynton CB, Chonde DB, Catana C. On the accuracy and reproducibility of a novel probabilistic atlasbased generation for calculation of head attenuation maps on integrated PET/MR scanners. Eur J Nucl Med Mol Imaging. 2016.

28. Magistretti PJ. Cellular bases of functional brain imaging: insights from neuron-glia metabolic coupling. Brain Res. 2000;886:108-12.

29. Yakushev I, Hammers A, Fellgiebel A, Schmidtmann I, Scheurich A, Buchholz HG, et al. SPM-based count normalization provides excellent discrimination of mild Alzheimer's disease and amnestic mild cognitive impairment from healthy aging. Neuroimage. 2009;44:43-50.

30. Guedj E, Bonini F, Gavaret M, Trébuchon A, Aubert S, Boucekine M, et al. 18FDG-PET in different subtypes of temporal lobe epilepsy: SEEG validation and predictive value. Epilepsia. 2015;56:414-21.

31. Antoine JC. Autoimmune encephalitis: paving the way for early diagnosis. Lancet Neurol. 2016;15:349-50. 